\author{
Organic and Medicinal Chemistry \\ International Journal \\ ISSN 2474-7610
}

\title{
Synthesis, In vitro Antibacterial and Antifungal Activities of Trifluoroalkyl-N, N'-Disubstituted Thioureas
}

\author{
Ines Chniti ${ }^{1}$, A Thebti ${ }^{2}$, M A K Sanhoury ${ }^{1,3 *}$, H I Ouzari Cherif ${ }^{2}$ and I Chehidi ${ }^{1}$ \\ ${ }^{1}$ Laboratory of Structural Organic Chemistry, Department of Chemistry, University of Tunis El-Manar, Tunisia \\ ${ }^{2}$ Laboratory of Microorganisms and Active Biomolecules (LMBA), Department of Biology, University of Tunis El Manar, Tunisia \\ ${ }^{3}$ Unité de Recherche en Chimie des Matériaux, Department of Chemistry, Mauritania
}

Submission: May 09, 2020; Published: July 06, 2020

*Corresponding author: MAK Sanhoury, Unité de Recherche en Chimie des Matériaux, Department of Chemistry. Faculty of Sciences and Techniques, USTM, Nouakchott, Mauritania

\begin{abstract}
A series of N-(4-trifluoromethylphenyl) thiourea derivatives of biological interest has been prepared by the condensation of various isothiocyanates with primary 4-trifluorophenylamine under uncatalyzed conditions. The chemical structures of all the reported compounds were confirmed by FT-IR, multinuclear NMR $(1 \mathrm{H}, 13 \mathrm{C}, 19 \mathrm{~F})$ and HRMS spectrometry. Some of these compounds were screened for their in vitro antibacterial activity against ten pathogenic strains representing different types of gram-positive and gram-negative bacteria, two pathogenic fungi (Penicilluim sp, Aspergillus flavus) and two yeasts (Candida albicans and Candida glabrata). More than four of the synthesized compounds showed promising inhibition activities against the tested strains Enterococcus faecuim ATCC 19436 with reference to standard vancomycin. Best antimicrobial activity was founded for the 3-pyridylthiourea derivative against Enterococcus faecuim ATCC 25923 with CMI $3.9 \mathrm{mg} / \mathrm{mL}$. The results indicated also that all the new screened compounds have promising antifungal activity comparable to the activity observed for the reference compounds.
\end{abstract}

Keywords: Isothiocyanates; Thioureas; Antibacterial activity; Antifungal activity

\section{Introduction}

This work is a continuation of our research on the study of sulfur having compounds in search of lead molecules [1-4]. In particulary isothiocyanates, wich are attractive synthons in organic chemistry due to their availability and their tendency additions and cycloadditions [5,6]. The strong electrophilicity of the NCS group of isothiocyanates enables these heterocumulenes to undergo nucleophilic addition and cycloaddition reactions, making them extremely useful in the synthesis of thiocarbamoyl derivatives [7]. Particularly isothiocyanates have been used for the synthesis of thioureas of synthetic, biological, agricultural and pharmaceutical interest [8-17]. Recently, thioureas have attracted considerable attention for their potential use as binding units for artificial receptors in supramolecular chemistry because of their characteristic behavior based on Lewis acids and strong hydrogen-bond donors [18-24].

Furthermore, in the field of advanced material chemistry, thioureas can serve as a useful scaffold by connecting them to electroluminescent organic dyes [25,26]. Thiourea group are also present in many drugs such as antithyroid, and anaesthetic [27]. Monothiourea derivatives, which are obtained by the condensation of isothiocyanate with esteramines, have shown strong antifungal activities, especially against Candida and Aspergillus in several studies [28-30]. Plaunotol and its thioureas derivatives presented antibacterial activities against Helicobater pylori as urease inhibitors [31,32]. Anti-HIV activity of thioureas were also reported in recent studies $[33,34]$. The literature survey reveals also that incorporation of halogen atom(s) within the molecule is one of the most effective strategies to enhance its biopotency, bioavailability and lipophilicity $[35,36]$.

Importantly, in recent studies fluorinated thioureas were also reported as novel class of potent influenza virus neuraminidase inhibitors $[37,38]$. Their enormous potential has led to the development of several methods for their preparation [27,39-41]. The most common of these methods involves the condensation of isothiocyanates with amino derivatives; Suresha et al. [42] 
proved that fluoro-containing arylthiourea compounds show better activity as compared to other analogues [42]. According to other authors findings [27,38,43], the antibacterial and antifungal efficiency depends on the presence of such electronwithdrawing substituent at C-2 and C-4 position of the phenyl ring. On the other hand, modification of isoxyl, the symmetrical diphenylthiourea derivative, produced the library of compounds with antimycobacterial activity [44].

To sum up, and as a part of the continuing research in our laboratory toward the development of the chemistry of new bioactive compounds containing fluoro groups under mild condition; we have previously disclosed the preparations of fluoroalkyl thiocarbamates and fluoroalkyl dithiocarbonates by the action of isothiocyanates and alcohols and thiols respectively $[1,2]$, and to investigate the role of the F-alkyl substitutant, the compounds were also evaluated for their antibacterial and antifungal activities [4], and the biological results were satisfactory and these laters proved to be good antibacterial and antifungal products.

Very recently, fluoro-methyl, or metoxy substituents on the 3-position benzene ring also improve antimicrobial potency $[45,46]$, however, and to the best our knowledge, the reaction of isothiocyanates with an amine having an F-alkylgroup at the para-position still remains much less explored. Here, we report the synthesis of new thioureas derivatives by condensation of isothiocyanates with 4-trifluoromethylphenyl, and testing for antifungal, antibacterial activities of these compounds. By using such amine, we want to combine antimicrobial effect of the F-alkyl group with the biological activity of thiourea group, together with the different $\mathrm{R}$ isothiocyanates groups (aliphatic, aromatic carbocyclic and heterocyclic groups, possessing electron withdrawing or electron-releasing groups).

\section{Materials and Methods}

\section{General}

Melting points (m.p.) were determined by using an Electrothermal 9100 apparatus and are uncorrected. Mass spectra were recorded on a high resolution Micromass micrOTOF-Q II 10027 spectrometer by electron spray ionization method. FTIR spectra were recorded on Nicolet IR200 spectrometer within the wave number range of $4000-400 \mathrm{~cm}^{-1}$ at $25^{\circ} \mathrm{C}$. Yields are of purified products. NMR spectra were recorded in the given solvent with Bruker AC300 spectrometer. Chemical shifts are reported as (values in parts per million) relative to TMS. The splitting pattern abbreviations are as follows: $m$ (multiple), s (singlet), $d$ (doublet), t (triplet). All reagents, solvents, and starting materials were obtained from commercial suppliers and used without further purification. Evaporations were conducted under reduced pressure. Reactions were monitored by thin layer chromatography (TLC) using Kieselgel 60 F254 (E. Merck) plates and UV detector for visualization. Flash column chromatography was performed on Silica Gel (70-230mesh).

\section{General experimental procedure for the synthesis of target compounds}

To a solution of the 4-trifluorophenylamine $(10 \mathrm{mmol})$ in $10 \mathrm{~mL}$ of THF, was added triethylamine $(15 \mathrm{mmol})$. Then the isothiocyanate $(10 \mathrm{mmol})$ was added. The mixture was Heat at the least for 2 hours. After vacuum evaporation of the solvent, the crude product was filter and purified either by chromatography (petroleum ether/diethyl ether (8:2) or by recrystallize from hexane or petroleum ether to give the corresponding pure compounds (1-9). We should note that the compound 7 has already been prepared by another experimental protocol in an earlier work [47] and the compounds 1, 3 and 8 are commercially available.

\section{3-3-Spectroscopic data}

$\mathrm{N}$-trifluoromethoxy-N'-trifluoromethylph enylthiourea $\mathrm{C}_{15} \mathrm{H}_{10} \mathrm{~F}_{6} \mathrm{~N}_{2} \mathrm{OS}(1)$<smiles>FC(F)(F)Oc1ccc(NC(=S)Nc2ccc(C(F)(F)F)cc2)cc1</smiles>

White solid m.p. $=131^{\circ} \mathrm{C}$; IR (KBr); $v\left(\mathrm{~cm}^{-1}\right) 3242(\mathrm{NH}), 3242$ $(\mathrm{NH}), 1086(\mathrm{C}=\mathrm{S}), \mathrm{RMN}^{1} \mathrm{H}\left(300 \mathrm{MHz}, \mathrm{CDCl}_{3}+\mathrm{DMSO}\right) \delta$ (ppm): 7.137.73 (syst $\mathrm{AB}, 8 \mathrm{H}, \mathrm{H}_{\text {arom }}$ ); 9.77 (broad signal $(2 \mathrm{NH})$ ). $\mathrm{RMN}{ }^{19} \mathrm{~F}(282$ $\left.\mathrm{MHz}, \mathrm{CDCl}_{3}+\mathrm{DMSO}\right) \delta(\mathrm{ppm}):-57.66\left(\mathrm{~s}, 3 \mathrm{~F}, \mathrm{O}-\mathrm{CF}_{3}\right) ;-61.57(\mathrm{~s}, 3 \mathrm{~F}$, CF). RMN ${ }^{13} \mathrm{C}\left(75 \mathrm{MHz}, \mathrm{CDCl}_{3}+\mathrm{DMSO}\right) \delta$ (ppm): 121.14; 123.11; 125.38; 125.61; 138.18; 142.83; 145.64 (8s, $\mathrm{C}_{\text {arom }}$ ); 180.16 (C=S). SMHR (ESI), m/z: cald for $\mathrm{C}_{15} \mathrm{H}_{10} \mathrm{~F}_{6} \mathrm{~N}_{2} \mathrm{OSH}^{+} 381.0475[\mathrm{M}+\mathrm{H}]^{+}$; found 381.0491.SMHR (ESI), $\mathrm{m} / \mathrm{z}$ : cald for $\mathrm{C}_{15} \mathrm{H}_{10} \mathrm{~F}_{6} \mathrm{~N}_{2} \mathrm{OSNa}^{+} 381.0475$ $[\mathrm{M}+\mathrm{H}]^{+}$; found 403.0310 .

$\mathrm{N}$-nitrophenyl-N'-trifluorome thylphenylthiourea $\mathrm{C}_{13} \mathrm{H}_{10} \mathrm{~F}_{3} \mathrm{~N}_{3} \mathrm{O}_{2} \mathrm{~S}(2)$<smiles>O=[N+]([O-])c1ccc(NC(=S)Nc2ccc(C(F)(F)F)cc2)cc1</smiles>

White solid; m.p. $=144^{\circ} \mathrm{C}$; IR (KBr); $v\left(\mathrm{~cm}^{-1}\right) 3242(\mathrm{NH}), 3242$ (NH), $1086(\mathrm{C}=\mathrm{S}) .{ }^{1} \mathrm{H}$ NMR (300 MHz, $\left.\mathrm{CDCl}_{3}+\mathrm{DMSO}\right) \delta(\mathrm{ppm}): 7.56-$ $8.17\left(\mathrm{~m}, 8 \mathrm{H}, \mathrm{H}_{\text {arom }}\right) .{ }^{19} \mathrm{~F}$ NMR $\left(282 \mathrm{MHz}, \mathrm{CDCl}_{3}+\mathrm{DMSO}\right) \delta(\mathrm{ppm})$ : $-61.81\left(\mathrm{~s}, 3 \mathrm{~F}, \mathrm{CF}_{3}\right) \cdot{ }^{13} \mathrm{C}$ NMR $\left(75 \mathrm{MHz}, \mathrm{CDCl}_{3}+\mathrm{DMSO}\right) \delta(\mathrm{ppm})$ : $121.96 ; 123.40 ; 124.16 ; 142.30 ; 143.07 ; 143.22 ; 145.55 ; 145.75$ $\left(\mathrm{C}_{\text {arom }}\right.$ ); $179.41(\mathrm{C}=\mathrm{S})$. HRMS (ESI), $\mathrm{m} / \mathrm{z}$ : cald for $\mathrm{C}_{14} \mathrm{H}_{10} \mathrm{~F}_{3} \mathrm{~N}_{3} \mathrm{O}_{2} \mathrm{SH}+$ $342.0519[\mathrm{M}+\mathrm{H}]^{+}$; found 342.0500. HRMS (ESI), $\mathrm{m} / \mathrm{z}$ : cald for $\mathrm{C}_{14} \mathrm{H}_{10} \mathrm{~F}_{3} \mathrm{~N}_{3} \mathrm{O}_{2} \mathrm{SNa}+364.0324[\mathrm{M}+\mathrm{Na}]^{+}$; found 364.0338. 
N-pyridyl-N'-trifluoromethylphenylthiourea $\mathrm{C}_{13} \mathrm{H}_{10} \mathrm{~F}_{3} \mathrm{~N} 3 \mathrm{~S}$ (3)<smiles>FC(F)(F)c1ccc(NC(=S)Nc2cccnc2)cc1</smiles>

White solid; m.p. $=146^{\circ} \mathrm{C}$; IR (KBr); $v\left(\mathrm{~cm}^{-1}\right) 3223(\mathrm{NH}), 3046$ $(\mathrm{NH}), 1596(\mathrm{C}=\mathrm{S}) . \mathrm{RMN}^{1} \mathrm{H}\left(300 \mathrm{MHz}, \mathrm{CDCl}_{3}+\mathrm{DMSO}\right) \delta$ (ppm): 7.29$7.33\left(\mathrm{~m},{ }^{1} \mathrm{H}\right) ; 7$ 7.56-7.77 (syst AB, $4 \mathrm{H}, \mathrm{H}_{\text {arom }}$ ); 8.07-8.10 (m, $\left.{ }^{1} \mathrm{H}\right) ; 8.31$ $8.35\left(\mathrm{~m},{ }^{1} \mathrm{H}\right) ; 8.60-8.64\left(\mathrm{~m},{ }^{1} \mathrm{H}\right) ; 9.80$ (broad signal $(\mathrm{NH})$ ); 9.97 (broad signal (NH)). RMN ${ }^{19} \mathrm{~F}\left(282 \mathrm{MHz}, \mathrm{CDCl}_{3}+\mathrm{DMSO}\right) \delta(\mathrm{ppm})$ : -61.65 (s, 3F, $\mathrm{CF}_{3}$ ). ${ }^{13} \mathrm{C}$ NMR (75 $\left.\mathrm{MHz}, \mathrm{CDCl}_{3}+\mathrm{DMSO}\right) \delta$ (ppm): $123.08 ; 123.27 ; 125.50 ; 125.55 ; 131.82 ; 136.01 ; 145.37 ; 145.60$ (9s, $\mathrm{C}_{\text {arom }}$ ); 180.49 (C=S). HRMS (ESI), m/z: cald for $\mathrm{C}_{13} \mathrm{H}_{10} \mathrm{~F}_{3} \mathrm{~N} 3 \mathrm{SH}+$ 298.0620 [M+H] ; found 298.0607. HRMS (ESI), m/z: cald for C13H10F3N3SNa+ $320.0440[\mathrm{M}+\mathrm{Na}]^{+}$; found 320.0420 .

$\mathrm{N}$-phenyl-N'-trifluoromethylphenylthiourea $\mathrm{C}_{14} \mathrm{H}_{11} \mathrm{~F}_{3} \mathrm{~N}_{2} \mathrm{~S}$ (4)<smiles>FC(F)(F)c1ccc(NC(=S)Nc2ccccc2)cc1</smiles>

White solid; m.p. $=152{ }^{\circ} \mathrm{C}$; IR (KBr); $v\left(\mathrm{~cm}^{-1}\right) 3203(\mathrm{NH}), 3030$ $(\mathrm{NH}), 1551(\mathrm{C}=\mathrm{S}) . \mathrm{NMR}{ }^{1} \mathrm{H}\left(300 \mathrm{MHz}, \mathrm{CDCl}_{3}+\mathrm{DMSO}\right) \delta(\mathrm{ppm})$ : 7.17-7.77 (m, 9H, $\mathrm{H}_{\text {arom }}$ ); 9.47 (broad signal $(2 \mathrm{NH})$ ). RMN ${ }^{19} \mathrm{~F}(282$ $\left.\mathrm{MHz}, \mathrm{CDCl}_{3}+\mathrm{DMSO}\right) \delta(\mathrm{ppm}):-61.84\left(\mathrm{~s}, 3 \mathrm{~F}, \mathrm{CF}_{3}\right) .{ }^{13} \mathrm{C} \mathrm{NMR}(75 \mathrm{MHz}$, $\left.\mathrm{CDCl}_{3}+\mathrm{DMSO}\right) \delta$ (ppm): 123.30; 123.56; 125.60; 125.64; 128.81; 138.52; 142.60 (8s, $\mathrm{C}_{\text {arom }}$ ); 180.01 (C=S). HRMS (ESI), m/z: cald for $\mathrm{C}_{14} \mathrm{H}_{11} \mathrm{~F}_{3} \mathrm{~N}_{2} \mathrm{SH}+297.0668[\mathrm{M}+\mathrm{H}]^{+}$; found 297.0659. HRMS (ESI) $\mathrm{m} / \mathrm{z}$ : cald for $\mathrm{C}_{14} \mathrm{H}_{11} \mathrm{~F}_{3} \mathrm{~N}_{2} \mathrm{SNa}+319.0487[\mathrm{M}+\mathrm{H}]^{+}$; found 319.0473 .

$\mathrm{N}$-benzyl-N'-trifluoromethylphenylthiourea $\mathrm{C}_{15} \mathrm{H}_{13} \mathrm{~F}_{3} \mathrm{~N}_{2} \mathrm{~S}$ (5)<smiles>FC(F)(F)c1ccc(NC(=S)NCc2ccccc2)cc1</smiles>

White solid; m.p. $=138^{\circ} \mathrm{C}$; IR (KBr); $v\left(\mathrm{~cm}^{-1}\right) 3299(\mathrm{NH}), 3047$ $(\mathrm{NH}), 1527(\mathrm{C}=\mathrm{S}) . \mathrm{NMR}{ }^{1} \mathrm{H}\left(300 \mathrm{MHz}, \mathrm{CDCl}_{3}\right) \delta(\mathrm{ppm}): 4.86(\mathrm{~d}$, $\left.2 \mathrm{H}, \mathrm{CH}_{2},{ }^{3} \mathrm{~J}_{\mathrm{H}-\mathrm{H}}=6.0 \mathrm{~Hz}\right) ; 6.46($ broad signal $(\mathrm{NH})) ; 7.29-7.64(\mathrm{~m}$, $5 \mathrm{H}, \mathrm{H}_{\text {arom. }}$ ); 8.55 (broad signal (NH)). NMR ${ }^{19} \mathrm{~F}\left(282 \mathrm{MHz}, \mathrm{CDCl}_{3}\right.$ ) $\delta$ (ppm): $-62.50\left(\mathrm{~s}, 3 \mathrm{~F}, \mathrm{CF}_{3}\right) .{ }^{13} \mathrm{C}$ NMR $\left(75 \mathrm{MHz}, \mathrm{CDCl}_{3}\right) \delta(\mathrm{ppm})$ : $49.54\left(\mathrm{~s}, \mathrm{CH}_{2}\right) ; 124.10 ; 127.81 ; 128.07 ; 128.96 ; 136.70 ; 139.66$ $\left(\mathrm{C}_{\text {arom }}\right)$; $180.50(\mathrm{C}=\mathrm{S})$. HRMS (ESI), m/z: cald for C15H13F3N2SH+ $311.0830[\mathrm{M}+\mathrm{H}]^{+}$; found 311.0821 .

$\mathrm{N}$-allyl-N'-trifluoromethylphenylthiourea $\mathrm{C}_{13} \mathrm{H}_{10} \mathrm{~F}_{3} \mathrm{~N}_{3} \mathrm{O}_{2} \mathrm{~S}(6)$<smiles>C=CCNC(=S)Nc1ccc(C(F)(F)F)cc1</smiles>

White solid; m.p. $=134^{\circ} \mathrm{C}$; IR (KBr); $v\left(\mathrm{~cm}^{-1}\right) 3265(\mathrm{NH}), 3077$ $(\mathrm{NH}), 1561(\mathrm{C}=\mathrm{S}) .{ }^{1} \mathrm{H}$ NMR $\left(300 \mathrm{MHz}, \mathrm{CDCl}_{3}\right) \delta(\mathrm{ppm}): 4.30(\mathrm{t}$, $\left.2 \mathrm{H}, \mathrm{CH}_{2},{ }^{3} \mathrm{~J}_{\mathrm{H}-\mathrm{H}}=6 \mathrm{~Hz}\right) ; 5.23\left(\mathrm{~m}, 2 \mathrm{H}, \mathrm{CH}_{2}\right) ; 5.90(\mathrm{~m}, 2 \mathrm{H},=\mathrm{CH}) ; 6.23$ (broad signal $(\mathrm{NH})$ ); 7.36-7.70 (syst $\mathrm{AB}, 4 \mathrm{H}, \mathrm{H}_{\text {arom }}$ ); 8.44 (broad signal (NH)). ${ }^{19} \mathrm{~F}$ NMR (282 MHz, $\mathrm{CDCl}_{3}$ ) $\delta(\mathrm{ppm}):-62.50(\mathrm{~s}, 3 \mathrm{~F}$, $\left.\mathrm{CF}_{3}\right) \cdot{ }^{13} \mathrm{C}$ NMR (75 MHz, $\left.\mathrm{CDCl}_{3}\right) \delta(\mathrm{ppm}): 47.97\left(\mathrm{~s}, \mathrm{CH}_{2}\right) ; 117.92(\mathrm{~s}$, $\left.\mathrm{CH}_{2}\right) ; 124.28 ; 127.33 ; 127.37 ; 139.68\left(4 \mathrm{~s}, \mathrm{C}_{\text {arom }}\right.$ ); $132.78(=\mathrm{CH})$; $180.61\left(\mathrm{C}=\mathrm{S}\right.$ ). HRMS (ESI), $\mathrm{m} / \mathrm{z}$ : cald for $\mathrm{C}_{9} \mathrm{H}_{13} \mathrm{~F}_{3} \mathrm{~N}_{2} \mathrm{SNa}+261.0649$ $[\mathrm{M}+\mathrm{Na}]^{+}$; found 261.0660 .

N-ethyl-N'-trifluoromethylphenylthiourea $\mathrm{C}_{10} \mathrm{H}_{11} \mathrm{~F}_{3} \mathrm{~N}_{2} \mathrm{~S}$ (7)<smiles>CCNC(=S)Nc1ccc(C(F)(F)F)cc1</smiles>

White solid; m.p. $=140^{\circ} \mathrm{C}$; IR (KBr); $v\left(\mathrm{~cm}^{-1}\right) 3268(\mathrm{NH}), 3088$ $(\mathrm{NH}), 1558(\mathrm{C}=\mathrm{S}) . \mathrm{NMR}^{1} \mathrm{H}\left(300 \mathrm{MHz}, \mathrm{CDCl}_{3}\right) \delta(\mathrm{ppm}): 1.23(\mathrm{t}, 3 \mathrm{H}$, $\left.\mathrm{CH}_{3},{ }^{3} \mathrm{~J}_{\mathrm{H}-\mathrm{H}}=6 \mathrm{~Hz}\right) ; 3.68\left(\mathrm{q}, 2 \mathrm{H}, \mathrm{CH}_{2}\right) ; 6.23$ (broad signal (NH)); 7.367.68 (syst $\mathrm{AB}, 4 \mathrm{H}, \mathrm{H}_{\text {arom. }}$ ); 8.83 (broad signal $(\mathrm{NH})$ ). NMR ${ }^{19} \mathrm{~F}(282$ $\mathrm{MHz}, \mathrm{CDCl}_{3}$ ) $\delta$ (ppm): -62.46 (s, 3F, $\left.\mathrm{CF}_{3}\right) .{ }^{13} \mathrm{C} \mathrm{NMR}\left(75 \mathrm{MHz}, \mathrm{CDCl}_{3}\right)$ $\delta$ (ppm): $14.16\left(\mathrm{~s}, \mathrm{CH}_{3}\right) ; 40.41\left(\mathrm{~s}, \mathrm{CH}_{2}\right) ; 124.07 ; 127.28 ; 127.44$; 139.92 (4s, $\mathrm{C}_{\text {arom }}$ ); $128.27\left(\mathrm{q}, \mathrm{CF}_{3},{ }^{1} \mathrm{~J}_{\mathrm{C}-\mathrm{F}}=20 \mathrm{~Hz}\right) ; 180.16(\mathrm{C}=\mathrm{S})$. HRMS (ESI), $\mathrm{m} / \mathrm{z}$ : cald for $\mathrm{C}_{10} \mathrm{H}_{11} \mathrm{~F}_{3} \mathrm{~N}_{2} \mathrm{SH}+249.0668[\mathrm{M}+\mathrm{H}]^{+}$; found 249.0665. HRMS (ESI), m/z: cald for $\mathrm{C}_{10} \mathrm{H}_{11} \mathrm{~F}_{3} \mathrm{~N}_{2} \mathrm{SNa}+271.0487$ $[\mathrm{M}+\mathrm{Na}]^{+}$; found 271.0478 .

N-butyl-N'-trifluoromethylphenylthiourea $\mathrm{C}_{12} \mathrm{H}_{15} \mathrm{~F}_{3} \mathrm{~N}_{2} \mathrm{~S}$ (8)<smiles>CCCCNC(=S)Nc1ccc(C(F)(F)F)cc1</smiles>

White solid; m.p. $=143^{\circ} \mathrm{C}$; IR (KBr); $v\left(\mathrm{~cm}^{-1}\right) 3268(\mathrm{NH}), 3088$ (NH), $1558(\mathrm{C}=\mathrm{S}) .{ }^{1} \mathrm{H}$ NMR (300 MHz, $\left.\mathrm{CDCl}_{3}\right) \delta(\mathrm{ppm}): 0.39(\mathrm{t}, 3 \mathrm{H}$, $\left.\mathrm{CH}_{3},{ }^{3} \mathrm{~J}_{\mathrm{H}-\mathrm{H}}=7 \mathrm{~Hz}\right) ; 1.35\left(\mathrm{~m}, 2 \mathrm{H}, \mathrm{CH}_{2}\right) ; 1.59\left(\mathrm{~m}, 2 \mathrm{H}, \mathrm{CH}_{2}\right) ; 3.63(\mathrm{q}, 2 \mathrm{H}$, $\mathrm{CH}_{2},{ }^{3} \mathrm{~J}_{\mathrm{H}-\mathrm{H}}=6 \mathrm{~Hz}$ ); 6.30 (broad signal $(\mathrm{NH})$ ); 7.37-7.67 (syst $\mathrm{AB}$, $4 \mathrm{H}, \mathrm{H}_{\text {arom. }}$ ); 8.78 (broad signal $(\mathrm{NH})$ ). ${ }^{19} \mathrm{~F} \mathrm{NMR}\left(282 \mathrm{MHz}, \mathrm{CDCl}_{3}\right) \delta$ (ppm): -62.45 (s, 3F, CF). ${ }^{13} \mathrm{C}$ NMR (75 MHz, $\mathrm{CDCl}_{3}$ ) $\delta$ (ppm): 13.76 (s, $\left.\mathrm{CH}_{3}\right) ; 20.16\left(\mathrm{~s}, \mathrm{CH}_{2}\right) ; 30.93\left(\mathrm{~s}, \mathrm{CH}_{2}\right) ; 45.32\left(\mathrm{~s}, \mathrm{CH}_{2}\right) ; 121.96$; 123.93; 127.03; 140.07 (4s, $\mathrm{C}_{\text {arom }}$ ); $128.15\left(\mathrm{q}, \mathrm{CF}_{3},{ }^{1} \mathrm{~J}_{\mathrm{C}-\mathrm{F}}=20 \mathrm{~Hz}\right.$ ); 
180.17 (C=S). HRMS (ESI), m/z: cald for $\mathrm{C}_{12} \mathrm{H}_{15} \mathrm{~F}_{3} \mathrm{~N}_{2} \mathrm{SH}+277.0981$ $[\mathrm{M}+\mathrm{H}]^{+}$; found 277.0976. HRMS (ESI), $\mathrm{m} / \mathrm{z}$ : cald for $\mathrm{C}_{12} \mathrm{H}_{15} \mathrm{~F}_{3} \mathrm{~N}_{2} \mathrm{SNa}+$ $299.0800[\mathrm{M}+\mathrm{Na}]^{+}$; found 299.0783.

$\mathrm{N}$ - cyclohexyl-N'-trifluorome thylphenylthiourea C13H10F $3 \mathrm{~N}_{3} \mathrm{O}_{2} \mathrm{~S}(9)$

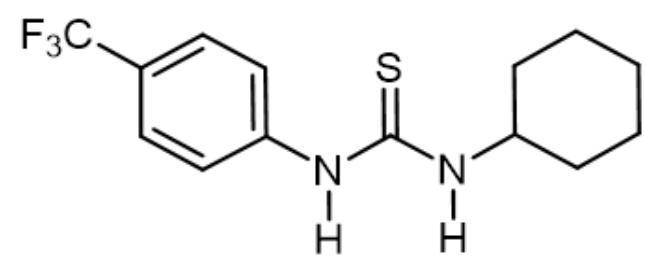

White solid; m.p. $=147^{\circ} \mathrm{C}$; IR (KBr); $v\left(\mathrm{~cm}^{-1}\right) 3269(\mathrm{NH}), 3046$ (NH), 1543 (C=S), RNM ${ }^{1} \mathrm{H}\left(300 \mathrm{MHz}, \mathrm{CDCl}_{3}\right.$ ) $\delta(\mathrm{ppm}): 1.20-2.11$ $\left(\mathrm{m}, 1^{1} \mathrm{H}\right) ; 6.15$ (d, NH); 7.35-7.67 (syst $\mathrm{AB}, 4 \mathrm{H}, \mathrm{H}_{\text {arom }}$ ); 8.49 (broad signal $(\mathrm{NH}))$. NMR ${ }^{19} \mathrm{~F}\left(282 \mathrm{MHz}, \mathrm{CDCl}_{3}\right) \delta(\mathrm{ppm}): 62.42(\mathrm{~s}, 3 \mathrm{~F}$, $\left.\mathrm{CF}_{3}\right)$. NMR ${ }^{13} \mathrm{C}\left(75 \mathrm{MHz}, \mathrm{CDCl}_{3}\right) \delta(\mathrm{ppm}): 24.72\left(\mathrm{CH}_{2}\right) ; 25.36\left(\mathrm{CH}_{2}\right)$; $32.50\left(\mathrm{CH}_{2}\right) ; 54.15(\mathrm{CH}) ; 123.75 ; 127.20 ; 127.24 ; 140.00(8 \mathrm{~s}$, $\mathrm{C}_{\text {arom }}$ ); $128.05\left(\mathrm{q}, \mathrm{CF}_{3}\right) ; 178.78(\mathrm{C}=\mathrm{S})$. HRMS (ESI), m/z: cald for $\mathrm{C}_{14} \mathrm{H}_{17} \mathrm{~F}_{3} \mathrm{~N}_{2} \mathrm{SH}+303.1137[\mathrm{M}+\mathrm{H}]^{+}$; found 303.1126. SMHR (ESI), $\mathrm{m} / \mathrm{z}$ : cald for $\mathrm{C}_{14} \mathrm{H}_{17} \mathrm{~F}_{3} \mathrm{~N}_{2} \mathrm{SNa}+325.0957[\mathrm{M}+\mathrm{Na}]^{+}$; found 325.0947 .

\section{Biological Essay}

\section{In vitro antibacterial evaluation}

The in vitro antibacterial activity was tested against five Gram positive strains (Enterococcus faecium ATCC 19436, Enterococcus faecalis ATCC 29212, Staphylococcus aureus ATCC 25923, Staphylococcus aureus ATCC 6539 and Bacillus cereus 49) and six Gram negative bacteria (Escherichia coli BLSE 3, Escherichia coli BLSE 10, Escherichia coli DH5a, Escherichia coli ATCC 8739, Pseudomonas aeruginosa and Salmonella $\mathrm{sp}$ ). The microdilution broth method according to recommendations of the National Committee for Clinical Laboratory Standards (NCCLS) [48] was used. Stock solutions of tested compounds in DMSO were twofold serially diluted to final concentrations ranging from 500 to $3.90 \mu \mathrm{g} / \mathrm{mL}$ in sterile 96 well microtiter plates which contained Mueller-Hinton Broth (MHB, Biolife Italiana S.r.I viale Monza, 272-20128 Milano, Italy). The bacterial cell density was kept uniformly throughout the experimentation at $1 \times 10^{8} \mathrm{CFU} / \mathrm{mL}$ by comparing with 0.5 McFrland turbidity standards and $100 \mu \mathrm{L}$ of test organisms was added in each well.

All procedures were performed in duplicate and microplates were incubated at optimal temperature and time $\left(37^{\circ} \mathrm{C}\right.$ for $24 \mathrm{~h}$, $30{ }^{\circ} \mathrm{C}$ for Bacillus cereus 49). Growth indicator (Dye A for Gram negative strains and Dye $G$ for positive ones $(100 \mu \mathrm{L}$ of $0.1 \%)$ were incorporated in each well to assess the bacterial inhibition. Well containing inoculum alone was used as negative control. Vancomycin was used as positive control. The minimum inhibitory concentrations (MICs) were assayed as a reduction in growth of at least $90 \%$ (IC90) as compared to the control. The MICs were determined and checked by a Biolog technologywhich enables high throughput automated kinetic cell assays. Biolog's Omnilog (BiologOmnilog ${ }^{\circledR}$ Phenotype MicroArray ${ }^{\mathrm{TM}}$ USA), Cell response in each assay well is determined by the amount of color development produced by the reduction of a tetrazolium compound (a redox Dye Mix) during cell respiration [49,50].

\section{In vitro antifungal evaluation}

The antifungal properties were evaluated in vitro against four fungal strains: Aspergillus flavus, Penicillium expansum, Candida albicans and Candida glabrata. The microdilution broth method was used according to NCCLS guidelines [51]. Stock solutions of tested compounds in DMSO were twofold serially diluted to final concentrations ranging from 500 to $3.90 \mu \mathrm{g} / \mathrm{mL}$ in sterile 96 well microtiter plates which contained Malt extracts (2\%). The fungal suspension turbidity was adjusted to $65 \%$ (104 conidia/mL) and $100 \mu \mathrm{l}$ was added to each well. All procedures were performed in duplicate and microplates were incubated at $28^{\circ} \mathrm{C}$ for $72 \mathrm{~h}$. Growth indicator (Dye E, $100 \mu \mathrm{L}$ of $0.1 \%$ ) was incorporated in each well. Wells containing inoculum alone was used as negative control. The minimum inhibitory concentrations (MICs) were analyzed visually and determined as the lowest concentrations for which there is no fungal growth then checked by the Biolog tool.

\section{Results and Discussion}

\section{Synthesis}

Our aim was to prepare a small library of new F-alkylated thiourea derivatives through a short synthetic method [52]. By choosing the appropriate precursors, the primary amine and the alkyl or aryl isothiocyanate, we can generate the chemical diversity of compounds. In the present study nine unsymmetrically $\mathrm{N}, \mathrm{N}$-disubstituted thioureas 1-9 were synthesized from commercially available 4-trifluoromethylphenylamine and a variety of substituted isothiocyanates. The reaction took place in one-step at refluxing THF and was completed in 1-2 hours in high yield (83-92\%) as shown on Scheme 1.

Different alkyl and aryl substituents of isothiocyanates were introduced to evaluate their effects on the biological activity of the compounds. Depending on these substituent group, a longer reaction period was needed in some cases to obtain the desired adducts and the results were summarized in Table 1 . That gives us a pool of compounds that, after screening process, will provide information about the structure-activity relationship. Table 1 shows the thiourea derivatives grouped by the type of lipophilic substituent. The structures were determined using different spectroscopic methods like multinuclear NMR, IR and HRMS spectra. 
Table 1: N-alkyl/aryl-N'-trifluoromethylphenylthiourea 1-9

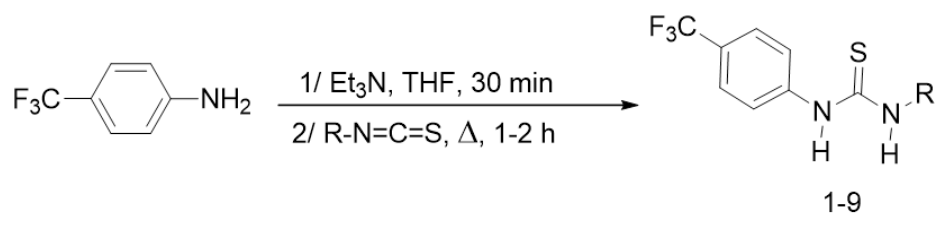

$(87-93) \%$

\begin{tabular}{|c|c|c|}
\hline Compound & R & Yield \\
\hline 1 & $4-\mathrm{CF}_{3} \mathrm{O}-\mathrm{Ph}$ & 93 \\
\hline 2 & $4-\mathrm{NO}_{2}-\mathrm{Ph}$ & 86 \\
\hline 3 & 3 -pyridyl & 89 \\
\hline 4 & Phenyl & 89 \\
\hline 5 & Benzyl & 83 \\
\hline 6 & Allyl & 90 \\
\hline 7 & Ethyl & 88 \\
\hline 8 & Butyl & 87 \\
\hline
\end{tabular}

\section{Characterization}

Spectral data (NMR, HRMS, IR) of all compounds were in full agreement with the proposed structures. The ${ }^{1} \mathrm{H}$ NMR spectrum exhibited singlets at $\delta 9.80-6.30 \mathrm{ppm}$, which were assigned to the $\mathrm{N}-\mathrm{H}$ protons. ${ }^{13} \mathrm{C}$ NMR revealed peaks, in the range of $178-$ 181ppm, for the typical signals for the thiocarbonylic carbons ( $\mathrm{C}=\mathrm{S}$ thiourea). Other ${ }^{13} \mathrm{C}$ NMR signals were considered a singlet if the multiplicity was not assigned. The very strong broad peak between 3200 and $3300 \mathrm{~cm}^{-1}$ on the FTIR spectrum, should be assigned to the extension vibration of the $\mathrm{N}-\mathrm{H}$ groups.

\section{Biological evaluation}

\section{Antibacterial activity}

Table 2: Antibacterial activity of thioureas, MIC $(\mu \mathrm{g} / \mathrm{mL})$.

\begin{tabular}{|c|c|c|c|c|c|}
\hline \multicolumn{6}{|c|}{ Gram Positive Bacteria } \\
\hline Compound & $E \cdot f^{a}$ & $E \cdot f^{b}$ & S. $a^{a}$ & S. $a^{b}$ & B.c \\
\hline 3 & 7.8 & 250 & 3.9 & 3.9 & 500 \\
\hline 4 & 7.8 & 250 & $\geq 500$ & $\geq 500$ & 125 \\
\hline 5 & 7.8 & 125 & $\geq 500$ & $\geq 500$ & 62.5 \\
\hline 6 & 15.62 & 250 & $\geq 500$ & 250 & 500 \\
\hline 7 & 250 & 500 & $\geq 500$ & $\geq 500$ & 250 \\
\hline Vancomycin & 125 & 62.5 & 3.9 & 31.25 & 0.39 \\
\hline
\end{tabular}

E.f: Enterococcus faecuim ATCC 19436, E.fo: Enterococcus faecalis ATCC 29212, B.c :Bacillus cereus 49, S.a: Staphylococcus aureus ATCC 6538, S.a $a^{b}$ : Staphylococcus aureus ATCC 25923.

\begin{tabular}{|c|c|c|c|c|c|c|}
\hline \multicolumn{7}{|c|}{ Gram Negative Bacteria } \\
\hline Compound & $E . c^{a}$ & $E . c^{b}$ & E. $c^{c}$ & $E . c^{d}$ & S.sp & P.a \\
\hline 3 & 250 & 125 & 250 & 500 & $>=500$ & 250 \\
\hline 4 & $\geq 500$ & 500 & $\geq 500$ & 500 & $\geq 500$ & $\geq 500$ \\
\hline 5 & $\geq 500$ & $\geq 500$ & 250 & 250 & $\geq 500$ & $\geq 500$ \\
\hline 6 & 500 & $\geq 500$ & 500 & $\geq 500$ & 7.81 & $\geq 500$ \\
\hline 7 & $\geq 500$ & $\geq 500$ & $\geq 500$ & $\geq 500$ & $\geq 500$ & $\geq 500$ \\
\hline Vancomycin & 125 & 62.5 & 250 & 250 & 125 & 250 \\
\hline
\end{tabular}

E.c $c^{a}$ : Escherichia coli DH5a, E.c ${ }^{b}$ : Escherichia coli ATCC 8739, E.c ${ }^{c}$ : Escherichia coli BLSE Aq 3, E.c $c^{d}$ : Escherichia coli BLSE Aq 10, S.sp : Salmonella sp, P.a : Pseudomonas aeruginosa 
Thioureas (1-9) (Table 1) were assayed in vitro against eleven bacterial strains: six are Gram-negative (Escherichia coli DH5 $\alpha$, Escherichia coli ATCC 8739, Escherichia coli BLSE Aq3, Escherichia coli BLSE Aq10, Pseudomonas aeruginosa and Salmonella sp) and five are Gram-positive (Enterococcus faecalis ATCC 29212, Enterococcus faecium ATCC 19436, Staphylococcus aureus ATCC 25923, Staphylococcus aureus ATCC6538 and Bacillus cereus 49) bacteria (Table 2). Gentamycin was used as reference drug for comparison purposes. The results showed that most of the compounds expressed moderate to excellent antibacterial activity (MIC values: $500-3.90 \mu \mathrm{g} / \mathrm{mL}$ ) not only towards typical Grampositive bacteria, but also towards Gram-negative bacteria.

Interestingly, all compounds were selectively more potent against Enterococcus faecuim ATCC 19436 than the reference drug vancomycin. The most substantial antibacterial profile was found for derivative 3 bearing electron withdrawing halogen atom on the phenyl ring, since they produced stronger electronegativity effect than it is observed for monosubstituted derivatives. These results are in accordance with our observations made for non-F-alkyl thiourea $[45,46,53,54]$. The presence of a nitrogen atom, at the aromatic ring is essential for a noticeable antimicrobial activity. However, the synthesized compounds have very weak effects on Escherichia coli DH5 $\alpha$ and Escherichia coli ATCC 8739. Thiourea compounds do not show the effect on the growth of Escherichia coli BLSE 3with MIC over than $500 \mu \mathrm{g} / \mathrm{mL}$.

Compound 3 show a light effect on Salmonella sp, Staphylococcus aureus 6539 and Staphylococcus aureus ATCC 25923 with MIC ranging between 3.9 and $7.8 \mathrm{mg} / \mathrm{mL}$; therefore, and in terms of structure-activity relationships (SARs), the potent antifungal activities are descending from the aryl groups and the alkyl moiety showed less effect on their antibacterial potency. The MICs of the antifungal activity of trifluoro phenyl thiourea derivatives against Aspergillus flavus and Penicilluim expansum is shown in Table 3. All derivatives showed significant in vitro antifungal activities against tested fungi. These compounds exhibited to have strong antifungal activities with low MICs values included in the range of 7.81-62.5 $\mu \mathrm{g} / \mathrm{mL}$.

Table 3: Antifungal activity of N-(4-(trifluoromethyl)-phenylt $\geq$ hiourea derivatives 1-9 MIC $(\mu \mathrm{g} / \mathrm{mL})$.

\begin{tabular}{|c|c|c|c|c|}
\hline \multirow{2}{*}{ Compound } & \multicolumn{2}{|c|}{ Fungi } & \multicolumn{2}{c|}{ Yeast } \\
\cline { 2 - 5 } & Aspergillus Flavus & Penicilluim Expansum & Candida Albicans & Candida Glabrata \\
\hline 3 & 31.25 & 31.25 & $\geq 500$ & 500 \\
\hline 4 & 31.25 & 31.25 & 500 & 500 \\
\hline 5 & 62.5 & 15.62 & 125 & 500 \\
\hline 6 & 31.25 & 15.62 & 125 & $\geq 500$ \\
\hline 7 & 7.81 & 15.62 & 2500 & 62.5 \\
\hline
\end{tabular}

a Values are the average of three reading.

In terms of structure-activity relationships (SARs), the potent antifungal activities are descending from the thiourea function and the R group. In general, Compounds 3, 4, 5, 6 and 7 exhibited stronger $(31.25-7.81 \mu \mathrm{g} / \mathrm{mL})$ or equal $(62.5 \mu \mathrm{g} / \mathrm{mL})$ antifungal activities than standard agent (Fluconazole). The results show that all the derivatives have effective activity against the tested fungi, compared with the standard Fluconazole, with MIC values ranging from 7.81 to $31.25 \mu \mathrm{g} / \mathrm{mL}$ against Aspergillus flavus and from 15.62 to $31.25 \mu \mathrm{g} / \mathrm{mL}$ against Penicilluim expansum according to the type of the derivative (Table 1 ). The results also showed that the substituent $\mathrm{R}$ (from the isothiocyanates employed in the reaction) plays a key role in varying the efficacy of antimicrobial activity.

Notably, the highest antifungal activity was observed for compound 7 (R=Ethyl) with MIC of $7.81 \mu \mathrm{g} / \mathrm{mL}$ against Aspergillus flavus and of $15.62 \mu \mathrm{g} / \mathrm{mL}$ against Penicilluim expansum. The compounds 3-6 exhibited to have strong antifungal activities with low MICs values included in the range of 31.25-62.5 $\mu \mathrm{g} /$ $\mathrm{mL}$ compared to the reference drug Fluconazole. However, the synthesized compounds have weak effects on Penicilluim expansum with MICs of $62.5 \mu \mathrm{g} / \mathrm{mL}$ and did not show the effect on the growth of Candida albicans ATCC 10231 and Candida glabrata with MIC over than $500 \mu \mathrm{g} / \mathrm{mL}$.

\section{Conclusion}

This paper reports the synthesis and the characterization of small libraries of trifluorophenyl-bisubstituted thioureas through an easy and high yielded reaction. We have also evaluated there in vitro antibacterial and antifungal properties. As can be seen from our results, most of these compounds found to be potent antibacterial and antifungal agent exhibited comparable to or even higher antibacterial and antifungal activity than the standard. Biological data revealed that the substituent R (from the isothiocyanates employed in the reaction) plays a key role in varying the efficacy of antimicrobial activity. Infact, the presence of an electron withdrawing group in the aromatic rings enhanced the antimicrobial activity of the synthesized compounds, showing in most cases more activity than that of the controls. The results presented in this work encourage us to continue in this line of research. 


\section{Funding}

This research was funded by the Tunisian Ministry of High Education and Scientific Research.

\section{References}

1. Chniti I, Sanhoury MAK, Chehidi I (2013) 0-perfluoroalkyl thiocarbamates: Synthesis and perfluoroalkyl effects on the barrier to NCS rotation. J Fluorine Chem 156: 101-105.

2. Chniti I, Sanhoury MAK, Merlet D, Chehidi I (2014) Synthesis and conformational study of new S-polyfluoroalkyl dithiocarbamates. J Fluorine Chem 168: 223-229.

3. Chniti I, Maouati H, Sanhoury MAK, Merlet D, Chehidi I (2017) Selective S-methylation of highly fluorinated thiocarbamates. Synth Commun 47: $15-21$

4. Thebti A, Chniti I, Sanhoury MAK, Chehidi I, Ouzari HI, et al. (2019) Antimicrobial activity of highly fluorinated thiocarbamates and dithiocarbamates. Curr Chem Biol 13 (2): 120-128.

5. Mukerjee AK, Ashare R (1991) Isothiocyanates in the chemistry of heterocycles. Chem Rev 91: 1-24.

6. Al Masoudi N, Hassan NA, Al Soud YA, Schmidt P, Gaafar ADM, et al. (1998) Syntheses of C- and N-nucleosides from 1-aza-2 azoniaallene and 1,3-diaza-2-azoniaallene salts. J Chem Soc Perkin Trans 1: 947954.

7. Maya I, Lopez O, Fernandez Bolanos JG, Robina I, Fuentes J (2001) A practical one-pot synthesis of 0 -unprotected glycosyl thioureas. Tetrahedron Letters 42: 5413-5416.

8. G Singh, A Saroa, S Rani, Promila, Shally Girdhar, et al. (2016) Substituted phenyl urea and thiourea silatranes: Synthesis, characterization and anion recognition properties by photophysical and theoretical studies. Polyhedron 112: 51-60.

9. Garcia Fernandez JM, Mellet CO (2000) Chemistry and developments of N-thiocarbonyl carbohydrate derivatives: Sugar isothiocyanates, thioamides, thioureas, thiocarbamates, and their conjugates. Adv Carbohydr Chem Biochem 55: 135-135.

10. Gasch C, Pradera MA, Salameh BAB, Molina JL, J Fuentes (2001) Isothiocyanato derivatives of sugars in the stereoselective synthesis of spironucleosides and spiro-C-glycosides. Tetrahedron: Asymmetry 12: 1267-1277.

11. Fernandez Bolanos J G, Zafra E, Lopez O, Robina I, Fuentes J (1999) Stereoselective synthesis of imidazolidine, imidazoline and imidazole C- and N-pseudonucleosides. Tetrahedron: Asymmetry10: 3011-3023.

12. Avalos M, Babiano R, Cintas P, MM Chavero, FJ Higes, et al (2000) Reactions of 2-Amino-2-thiazolines with Isocyanates and Isothiocyanates. Chemical and Computational Studies on the Regioselectivity, Adduct Rearrangement, and Mechanistic Pathways. J Org Chem 65: 8882-8892.

13. Herr RJ, Kuhler JL, Meckler H, CJ Opalka (2000) Convenient Method for the Preparation of Primary and Symmetrical N, N'-Disubstituted Thioureas. J Synthesis 2000 (11): 1569-1574.

14. Venkatachalam TK, Sudbeck EA, Uckun FM (2001) Regiospecific synthesis, X-ray crystal structure and biological activities of 5-bromothiophenethyl thioureas. Tetrahedron Lett 42: 6629-6632.

15. Todoulou OG, Papadaki Valiraki AE, Filippatos EC, Ikeda S, De Clercq E (1994) Synthesis and anti-myxovirus activity of some novel N, N'disubstituted thioureas. Eur J Med Chem 29: 127-131.

16. Roy R (1996) Syntheses and some applications of chemically defined multivalent glycoconjugates. Curr Opin Struct Biol 6: 692-702.
17. Asghar F, Lal B, Badshah A, Butler IS, Tahir MN (2019) Synthesis and computational study of new meta- and para-substituted ferrocenyl thioureas as potent protein kinase inhibitors and cytotoxic agents. Inorganica Chimica Acta 488: 8-18.

18. Snellink Ruel BHM, Antonisse MMG, Engbersen JFJ, Timmerman P, Reinhoudt DN (2000) Neutral Anion Receptors with Multiple UreaBinding Sites. Eur J Org Chem 2000: 165-170.

19. Sasaki S, Mizuno M, Naemura K, TobeY (2000) Synthesis and AnionSelective Complexation of Cyclophane-Based Cyclic Thioureas. J Org Chem 65: 275-283.

20. Tozawa T, Misawa Y, Tokita S, Kubo Y (2000) A regioselectively bis(thiourea)-substituted dibenzo-diaza-30-crown-10: a new strategy for the development of multi-site receptors. Tetrahedron Lett 41: 5219-5223.

21. Lee KH, Hong JI (2000) C3-Symmetric metacyclophane-based anion receptors with three thiourea groups as linkers between aromatic groups. Tetrahedron Lett 41: 6083-87.

22. Lee DH, Lee KH, Hong JI (2001) An Azophenol-Based Chromogenic Anion Sensor. Org Lett 3: 5-8.

23. Benito JM, Gomez Garcıa M, Jimenez Blanco JL, Gómez García M, Mellet CO (2001) Carbohydrate-Based Receptors with Multiple Thiourea Binding Sites. Multipoint Hydrogen Bond Recognition of Dicarboxylates and Monosaccharides. J Org Chem 66: 1366-1372.

24. Boas U, Karlsson AJ, de Waal BFM, EW Meijer (2001) Synthesis and Properties of New Thiourea-Functionalized Poly(propylene imine) Dendrimers and Their Role as Hosts for Urea Functionalized Guests. J Org Chem 66: 2136-2145.

25. Werts MHV, Woudenberg RH, Emmerink PG, van Gassel, R, Hofstraat JW, et al. (2000) A Near-Infrared Luminescent Label Based on YbII Ions and Its Application in a Fluoroimmunoassay. Angew Chem Int Ed Engl 39: 4542-4544.

26. Ueda M, Sawai Y, Yamamura S (2000) Syntheses of fluorescence-labeled artificial leaf-opening substances, fluorescent probe compounds useful for bioorganic studies of nyctinasty. Tetrahedron Lett 41: 3433-3436.

27. Satchell DPN, Satchell RS (1993) The Chemistry of Sulfur-Containing Functional Groups. In: Patai S, Rappoport Z (Eds.), Wiley: New York, Suppl. S, Chapter 12, pp. 599-631.

28. Caujolle R, Amarouch H, Payard M Loiseau PM, Bories C, et al. (1995) Synthesis, antifungal and nematocidal activities of thioureines with an aminoester sequence. Eur J Med Chem 30: 801-807.

29. Truong P, Ngo DTH (1999) J Vietnamese Pharm 12. Chem. Abstr 2000 133: $263-752$.

30. Truong P, Tran PY (2000) J Vietnamese Pharm 9. Chem Abstr 2001135 73923.

31. Hiroshi K, Keiko T, Masami A, Minami E, Masuda K, et al (1999) A highly stereoselective synthesis of plaunotol and its thiourea derivatives as potent antibacterial agents against Helicobacter pylori. Bioorg Med Chem Lett 9: 1347-1350.

32. Demuth Jr, Thomas P, White RE (1997) United States patent US 5 631: 256.

33. Uckun FM, Mao C, Pendergrass S, Maher D, Zhu D et al (1999) N-[2(1-cyclohexenyl)ethyl]-N'-[2-(5-bromopyridyl)]-thiourea and $\mathrm{N}^{\prime}-[2-$ (1-cyclohexenyl)ethyl]- $\mathrm{N}^{\prime}$-[2-(5-chloropyridyl)]-thiourea as potent inhibitors of multidrug-resistant human immunodeficiency virus-1. Bioorg Med Chem Lett 9: 2721-2726.

34. Dong Y, Venkatachalam TK, Narla RK, Trieu VN, Sudbeck EA, et al (2000) Antioxidant function of phenethyl-5-bromo-pyridyl thiourea compounds with potent anti-HIV activity. Bioorg Med Chem Lett 10: 87-90. 
35. Ehrhard AA, Jäger S, Malm C, Basaran S, Hunger J (2019) CF3-groups critically enhance the binding of thiourea catalysts to ketones - a NMR and FT-IR study. Journal of Molecular Liquids $296: 111829$

36. Rainer W, Zimmermann MO, Lange A, Joerger AC, Boeckler FM (2012) Principles and Applications of Halogen Bonding in Medicinal Chemistry and Chemical Biology. J Med Chem 1363-1388.

37. Ghorab MM, Alsaid MS, El Gaby MSA, Elaasser MM, Nissan YM (2017) Antimicrobial and anticancer activity of some novel fuorinated thiourea derivatives carrying sulfonamide moieties: synthesis, biological evaluation and molecular docking. Chemistry Central Journal 11: 32 46.

38. Maalik A, Rahim H, Saleem M, Fatima N, Rauf A, et al. (2019) Synthesis, antimicrobial, antioxidant, cytotoxic, antiurease and molecular docking studies of $\mathrm{N}-(3$ trifluoromethyl) benzoyl-N'-aryl thiourea derivatives. Bioorganic Chemistry 88: 102946-102955.

39. Wahid S, Jahangir S, Versiani MA, Khan KM, Salar U, et al. (2020) Atenolol Thiourea Hybrid as Potent Urease Inhibitors: Design, BiologyOriented Drug Synthesis, Inhibitory Activity Screening, and Molecular Docking Studies. Bioorganic Chemistry 94: 103359-103367.

40. Al Harbi RAK, El Sharief MAM Sh, Abbas SY (2019) Synthesis and anticancer activity of bis-benzo[d] [1,3]dioxol-5-yl thiourea derivatives with molecular docking study. Bioorganic Chemistry 90:103088.

41. Saeed A, Shaheen U, Hameed A, Haider Naqvi SZ (2009) Synthesis, characterization and antimicrobial activity of some new 1-(fluorobenzoyl)-3-(fluorophenyl)thioureas. J Fluorine Chem 130: 1028-1034

42. Suresha GP, Suhas R, Kapfo W, D Channe Gowda (2011) Urea/thiourea derivatives of quinazolinone-lysine conjugates: Synthesis and structure-activity relationships of a new series of antimicrobials. Eur J Med Chem 46: 2530-2540.

43. Chikhalia KH, Patel MJ (2009) Design, synthesis and evaluation of some 1,3,5-triazinyl urea and thiourea derivatives as antimicrobial agents. J Enzyme Inhib Med Chem 24: 960-966.

44. Saeed S, Rashid N, Jones PG, Ali M, Hussain R (2010) Synthesis, characterization and biological evaluation of some thiourea derivatives bearing benzothiazole moiety as potential antimicrobial and anticancer agents. Eur J Med Chem 45: 1323-1331.
45. A Bielenica K Stępień, A Napiórkowska, E Augustynowicz Kopeć, S Krukowski, et al. (2016) Synthesis and Antimicrobial Activity of 4Chloro-3-Nitrophenylthiourea Derivatives Targeting Bacterial Type II Topoisomerases. Chem Biol Drug Des 87: 905-917.

46. Bielenica A, Stefańska J, Stępień K, Napiórkowska A, Augustynowicz Kopeć E (2015) Synthesis, cytotoxicity and antimicrobial activity of thiourea derivatives incorporating 3-(trifluoromethyl)phenyl moiety. Eur J Med Chem 101: 111-125.

47. Duke RM, Gunnlaugsson T (2007) Selective fluorescent PET sensing of fluoride (F-) using naphthalimide-thiourea and -urea conjugates. Tetrahedron Letters 48: 8043-8047.

48. National Committee for Clinical Laboratory Standards (2000) Methods for dilution antimicrobial susceptibility tests for bacteria that grow aerobically. Approved standard M7-A4. National Committee for Clinical Laboratory Standards, Wayne, Pennsylvania.

49. Bochner BR, Gadzinski P, Panomitros E (2001) Phenotype microarrays for high-throughput phenotypic testing and assay of gene function. Genome Res 11: 1246-1255.

50. Bochner BR (2003) New technologies to assess genotype-phenotype relationships. Nat Rev Genet 4 (4): 309-314.

51. Clinical Laboratory Standards Institute (2008) Reference method for Broth Dilution Antifungal Susceptibility Testing of Yeasts, Approved Standard ( $3^{\text {rd }}$ edn), M27-A3, Wayne, Pennsylvania.

52. Kumamoto K, Misawa Y, Tokita S, Kubo Y, Kotsuki H (2002) High-pressure-promoted condensation of isothiocyanates with aminopyridines: efficient synthesis of pyridine-thiourea conjugates as building blocks for hydrogen-bonding receptors. Tetrahedron Lett 43: 1035-1038.

53. Bhowruth V, Brown AK, Reynolds RC, Coxon GD, Mackay SP, et al. (2006) Symmetrical and unsymmetrical analogues of isoxyl; active agents againstMycobacterium tuberculosis. Bioorg Med Chem Lett 1647: 4743-4747.

54. Faidallah HM, Rostom SA, Basaif SA, Makki MS, Khan KA (2013) Synthesis and biological evaluation of some novel urea and thiourea derivatives of isoxazolo[4,5-d] pyridazine and structurally related thiazolo[4,5-d] pyridazine as antimicrobial agents. Arch Pharm Res 36: 1354-1368.

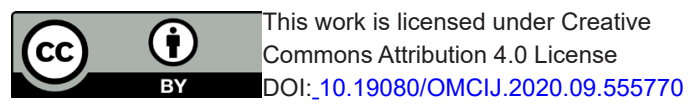

Your next submission with Juniper Publishers
will reach you the below assets
- Quality Editorial service
- Swift Peer Review
- Reprints availability
- E-prints Service
- Manuscript Podcast for convenient understanding
- Global attai nment for your research
- Manuscript accessibility in different formats
( Pdf, E-pub, Full Text, Audio)
- Unceasing customer service
Track the below URL for one-step submission
https://juniperpublishers.com/online-submission.php

